\title{
BMJ Open Cardiovascular disease risk marker responses to breaking up prolonged sedentary time in individuals with paraplegia: the Spinal Cord Injury Move More (SCIMM) randomised crossover laboratory trial protocol
}

Thomas M Withers, ${ }^{1}$ Louise Croft, ${ }^{1}$ Victoria L Goosey-Tolfrey, ${ }^{2}$ David W Dunstan, ${ }^{3,4}$ Christof A Leicht, ${ }^{2}$ Daniel P Bailey ${ }^{1}$

To cite: Withers TM, Croft L, Goosey-Tolfrey VL, et al. Cardiovascular disease risk marker responses to breaking up prolonged sedentary time in individuals with paraplegia: the Spinal Cord Injury Move More (SCIMM) randomised crossover laboratory trial protocol. BMJ Open 2018;8:e21936. doi:10.1136/ bmjopen-2018-021936

- Prepublication history and additional material for this paper are available online. To view these files, please visit the journal online (http://dx.doi. org/10.1136/bmjopen-2018021936).

Received 26 January 2018

Revised 16 May 2018

Accepted 24 May 2018
Check for updates

For numbered affiliations see end of article.

Correspondence to

Dr Daniel P Bailey;

daniel.bailey@beds.ac.uk

\section{ABSTRACT}

Introduction Sedentary behaviour is a distinct risk factor for cardiovascular disease (CVD) and could partly explain the increased prevalence of CVD in people with spinal cord injury (SCl). Interrupting prolonged sitting periods with regular short bouts of walking acutely suppresses postprandial glucose and lipids in able-bodied individuals. However, the acute CVD risk marker response to breaking up prolonged sedentary time in people with $\mathrm{SCl}$ has not been investigated.

Methods and analysis A randomised two-condition laboratory crossover trial will compare: (1) breaking up prolonged sedentary time with 2 min moderate-intensity arm-crank activity every $20 \mathrm{~min}$, with (2) uninterrupted prolonged sedentary time (control) in people with SCl. Outcomes will include acute effects on postprandial glucose, insulin, lipids and blood pressure. Blood samples will be collected and blood pressure measured at regular intervals during each $5 \frac{1}{2} 2$-hour condition.

Ethics and dissemination This study was approved by the Cambridge South National Health Service Research Ethics Committee. This research will help determine if breaking up prolonged sedentary time could be effective in lowering CVD risk in people with SCI. The findings of the research will be published in a peer-reviewed journal and disseminated to relevant user groups.

Trial registration number ISRCTN51868437; Pre-results.

\section{INTRODUCTION}

There is a global incident rate of 180000 traumatic spinal cord injury (SCI) cases each year with a prevalence of over 40000 in the UK. ${ }^{12}$ Cardiovascular disease (CVD) is a leading cause of death in individuals with $\mathrm{SCI}^{3}{ }^{3}$ and this population has a significantly increased risk of heart disease and stroke compared with able-bodied individuals. ${ }^{4}$ Traditional risk factors for CVD include impaired glucose tolerance, central obesity,

\section{Strengths and limitations of this study}

- This study uses a randomised crossover design to investigate, for the first time, cardiovascular disease risk marker responses to breaking up prolonged sedentary time in individuals with paraplegia.

- Regular collection of blood samples will permit robust time course and incremental area under the curve calculations for primary and secondary outcomes.

- Due to the acute nature of the study, the long-term cardiovascular disease risk marker responses to a chronic intervention will remain unknown.

- The cardiovascular disease risk marker responses to breaking up prolonged sedentary time in people with tetraplegia still requires investigation.

high triglycerides, low high-density lipoprotein cholesterol (HDL) and high blood pressure. These risk factors often exacerbate significantly as a consequence of $\mathrm{SCI},{ }^{5}$ and a plethora of research has documented impaired glucose tolerance and adverse lipid profiles in individuals with SCI. ${ }^{5}$ The clustering of $\geq 2$ and $\geq 3$ risk factors is prevalent in $87 \%$ and $72 \%$ of SCI individuals, respectively, ${ }^{7}$ which is markedly higher compared with the able-bodied population. ${ }^{8}$ This milieu of metabolic disturbances after SCI may be due to increases in body fat resulting from an imbalance in energy intake and expenditure. ${ }^{5}$ Excess fat accumulation, particularly in the visceral region, is associated with inflammation that is causal in glucose intolerance and dyslipidaemia, ${ }^{59}$ thus promoting atherogenesis that would increase the risk of CVD in this population. ${ }^{10}$ 
Postprandial glucose and lipid concentrations are strong independent predictors of future CVD incidence, even in those without diabetes. ${ }^{11}$ There is a doseresponse relationship between postprandial glucose area under the curve (AUC) and CVD risk, while progression of carotid atherosclerosis can be prevented by attenuation of postprandial glucose concentrations. ${ }^{12}{ }^{13}$ Impaired postprandial glucose metabolism was observed in 50\% and $62 \%$ of individuals with paraplegia and tetraplegia, respectively, compared with $18 \%$ in able-bodied individuals. ${ }^{6}$ This impaired glucose tolerance in SCI is characterised by hyperinsulinaemia, which suggests that there is tissue-level resistance to insulin. ${ }^{14}$ In individuals with paraplegia, there appears to be no difference in postprandial glucose responses between those with complete versus incomplete lesions. ${ }^{15}{ }^{16}$ Although postprandial lipaemic responses have not been compared between individuals with complete and incomplete lesions, fasting lipid levels do not differ between these groups. ${ }^{17}$ There does, however, appear to be an exaggerated postprandial lipaemic response in individuals with paraplegia compared with able-bodied individuals. ${ }^{18}$ These observations are of potential concern as the high dietary intake of carbohydrate and fat in individuals with $\mathrm{SCI}^{19}$ may lead to repeated exaggerated elevations in glucose and lipids following food intake. It is thus pertinent to identify interventions to reduce postprandial glucose and lipid responses in individuals with SCI to reduce their CVD risk.

Physical activity guidelines have been developed specifically for the SCI population that recommend engaging in at least $30 \mathrm{~min}$ of moderate-to-vigorous physical activity (MVPA) three times per week for CVD health benefits. ${ }^{20}$ However, it is estimated that $37 \%-50 \%$ of this population engage in no leisure-time physical activity whatsoever. ${ }^{21} 22$ Reduced levels of physical activity are proposed to largely account for the increased CVD risk in SCI with reduced levels of leisure-time physical activity associated with increased body fat, insulin resistance and systolic blood pressure. ${ }^{2223}$ However, sedentary behaviour (ie, any waking behaviour in a sitting, reclining or lying posture with low energy expenditure $)^{24}$ is now recognised as being a significant CVD risk factor in the able-bodied population, independent of MVPA. ${ }^{25}$ Experimental studies in able-bodied individuals have reported an acute reduction in postprandial glucose, insulin, triglycerides and blood pressure in response to breaking up prolonged sedentary time with $2 \mathrm{~min}$ bouts of light or moderate-intensity walking every 20 min. ${ }^{26-29}$ However, no research has examined whether postprandial CVD risk marker responses are attenuated in response to breaking up prolonged sedentary time in individuals with SCI.

The primary aim of this study is therefore to compare the acute CVD risk marker responses in individuals with SCI to (1) breaking up prolonged sedentary time, with (2) uninterrupted sedentary time. The CVD risk markers that will be studied include postprandial glucose (primary outcome), insulin and lipids, and systolic and diastolic blood pressures (secondary outcomes) based on evidence that these markers predict CVD outcomes and are adversely affected by SCI. It is hypothesised that breaking up prolonged sedentary time will result in favourable CVD risk marker responses compared with uninterrupted sedentary time in individuals with paraplegia. This could identify a novel strategy for the prevention of CVD in SCI that would warrant further evaluation.

\section{METHODS AND ANALYSIS \\ Study design}

A randomised two-condition crossover design will be used in accordance with the Standard Protocol Items: Recommendations for Interventional Trials (SPIRIT) statement. ${ }^{30}$ The study schedule can be seen in figure 1 . All research will take place at the University of Bedfordshire Sport and Exercise Science Laboratories. After preliminary measures, participants will complete two experimental conditions in a randomised order. The conditions will be separated by $\geq 6$ days to eliminate any potential carry-over effects. Condition order will be randomised by a researcher independent from the study using computer-generated random numbers (block randomisation with balanced block sizes).

\section{Participants}

\section{Inclusion criteria}

Males and females aged 18-60 years; chronic SCI ( $\geq 1$ year since injury), individuals with a traumatic SCI below T5

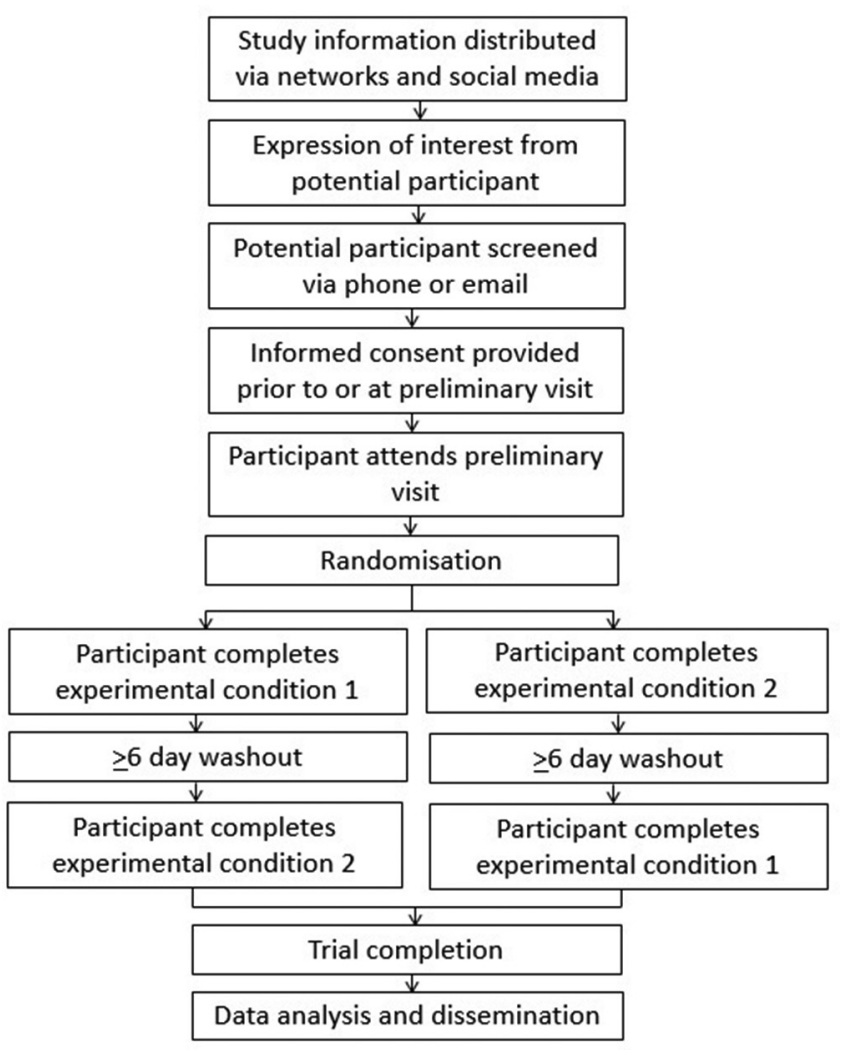

Figure 1 Study schedule. 
(mid to low level paraplegia), individuals with a non-traumatic SCI (as defined by the International Spinal Cord Injury Data Sets for non-traumatic $\mathrm{SCI}^{31}$ ) that present with mid to low level paraplegia. Including only individuals with injuries below $\mathrm{T} 5$ will ensure sympathetic innervation to the major organs at the T5 level so that heart rate and catecholamine responses would be unaffected by injury ${ }^{32}$ and thus minimise the potential that innervation variations could have on the study outcomes. Individuals with paraplegia who have complete or incomplete lesions will be included based on evidence that these groups do not differ with respect to postprandial glucose metabolism (primary outcome) ${ }^{15}{ }^{16}$ Individuals who express an interest in taking part in the study will be required to indicate their spinal cord lesion level and completeness of injury via a questionnaire and asked to provide the research team with a copy of medical records to confirm injury level and American Spinal Injury Association (ASIA) impairment scale classification prior to preliminary measures.

\section{Exclusion criteria}

Individuals who regularly engage in $>300 \mathrm{~min} /$ week of MVPA as such high levels of physical activity may offset the detrimental association of sedentary time with health outcomes $^{33}$; history of severe cardiovascular complications; hypotension (resting blood pressure $<90 / 60 \mathrm{~mm}$ $\mathrm{Hg}$ ); body mass index $>45 \mathrm{~kg} / \mathrm{m}^{2}$; a history of autonomic dysreflexia; pregnancy; taking glucose-lowering medication; smokers; diagnosed diabetes, renal failure, liver disease, major illness or other health issues that may limit the ability to perform the physical activity protocols.

\section{Recruitment}

Participants will be recruited through organisations and charities relevant to individuals with SCI, including the National Spinal Injuries Centre, Stoke Mandeville Hospital, Buckinghamshire NHS Healthcare Trust; local sport and activity clubs; and local community groups. Mail outs, social media, information on websites, posters, flyers and visits from the research team will be used to provide information on the study to potentially eligible individuals who can then express their interest to the research team in taking part in the study. Written informed consent will be obtained by a member of the research team prior to participation in any testing protocols (see online supplementary file). As an incentive, participants will receive a $£ 25$ shopping gift voucher for each main condition they complete and will have all travel expenses paid.

\section{Preliminary measures}

Participants will attend a preliminary testing session where they will have body mass measured using wheelchair double beam scales (300 series; Marsden, London, UK). They will also have body fat and lean tissue mass (and per cent) determined for the whole body and regionally via whole-body scans using dual-energy X-ray absorptiometry (DXA; GE Medical Systems; Chalfont
St Giles, UK) in line with previous research. ${ }^{34-36}$ During DXA measures, participants will be positioned as closely as possible to standard protocols, and Velcro restraints will be fastened around the participants' knees and ankles to maintain correct position of the legs during scanning. Participants will be offered a wedge to be used as a pillow for comfort. Waist circumference will be measured using the International Society for the Advancement of Kinanthropometry (ISAK) International Standards for Anthropometric Assessment guidelines. ${ }^{37} 38$ These measures will be taken in the standing position for participants who are able to maintain this posture and in a supine position for participants who are not able to stand. ${ }^{38}$ Resting blood pressure will be measured on the left arm, while seated, three times after the participant has rested for 5 min with the lowest readings being recorded. Following this, participants will be familiarised with use of the Borg 6-20 Rating of Perceived Exertion (RPE) scale. ${ }^{39}$ They will then cycle using an arm ergometer (Lode Angio; Lode, Netherlands) to determine the intensity (power output) that yields an RPE of 13 (somewhat hard) in line with previous sedentary behaviour research. ${ }^{26}{ }^{40}$ Participants will be asked to cycle at $\sim 70 \mathrm{rpm}$ during the test. The test will start at a low intensity ( 20 Watts) and the participants will then indicate their RPE at 1 min intervals. The resistance will then be increased by $5-20$ Watts based on the participants' RPE until an RPE of 13 has been achieved, at which point the test will be terminated. The test is expected to take no longer than $15 \mathrm{~min}$. The intensity that corresponds to an RPE of 13 during the test will be recorded for each participant and used for the physical activity breaks described in the respective main condition below. The use of the Borg 6-20 RPE scale has acceptable validity in individuals with SCI to determine physical activity intensity. ${ }^{41}$ This method is also suggested as a practical approach for healthcare professionals and scientists as oxygen consumption testing equipment is costly and not available in many rehabilitation centres and community settings. ${ }^{41}$

\section{Experimental protocol}

Figure 2 shows the experimental protocol. Participants will be instructed to refrain from caffeine, alcohol and exercise for 48 hours prior to each experimental condition. They will also be provided with a food diary and digital weighing scales to record volume and timings of all food and liquids consumed in the 24-hour period prior to the first experimental condition. Participants will be asked to replicate their diet the day prior to the subsequent experimental condition. ${ }^{42}$ On condition days, participants will attend in the morning following an overnight fast and avoid active travel to the laboratory. On arrival, resting blood pressure will be measured after $5 \mathrm{~min}$ rest; two measures will be taken and the lowest of these recorded. A fasting capillary blood sample will then be collected. Participants will commence the $5^{1 / 2}$-hour condition period following consumption of a standardised breakfast. The two experimental conditions are as follows: 


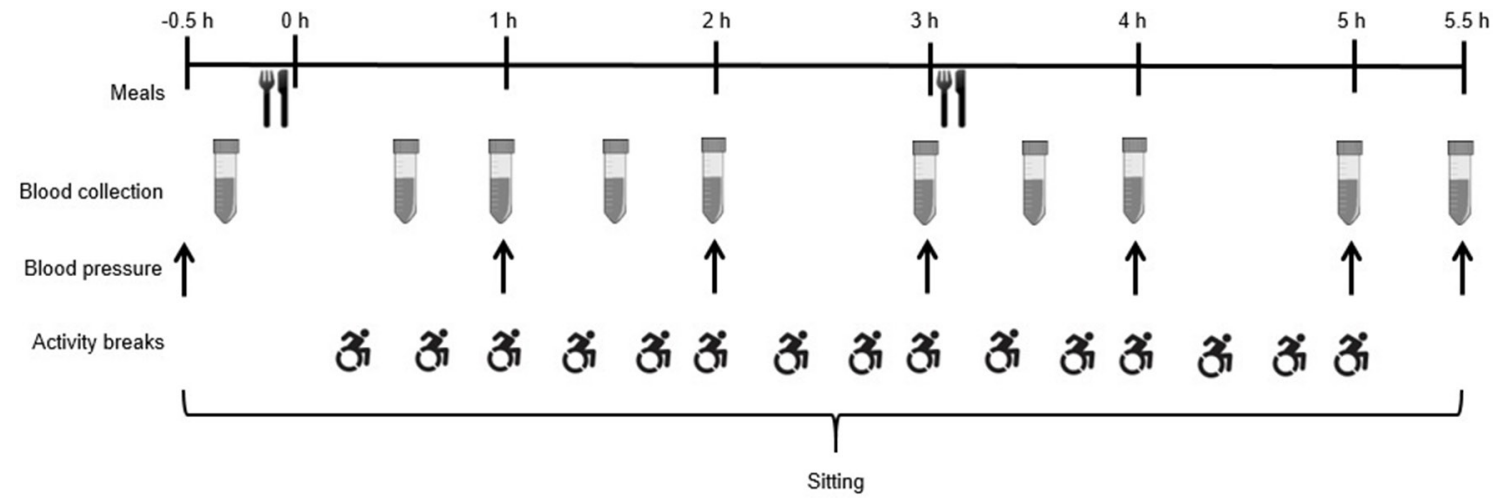

Figure 2 Schematic of experimental protocol.

1. Uninterrupted sedentary time (SED): participants will remain seated and inactive in their wheelchair or a standard chair at a desk during this condition.

2. Sedentary time interrupted with physical activity breaks (SED$A C T$ ): participants will complete 2 min of moderate-intensity arm-crank activity every $20 \mathrm{~min}$ at $\sim 70 \mathrm{rpm}$ using the Lode Angio arm ergometer. These 15 breaks will equate to a total of 30 min physical activity.

The SED-ACT protocol was selected based on previous research that reported a significant reduction in 5-hour postprandial glucose in response to breaking up prolonged sitting time with 2 min light-intensity walking every $20 \mathrm{~min}$ versus uninterrupted sitting in able-bodied participants. ${ }^{28}$ An RPE of 13 for the physical activity intensity was selected in line with previous research, ${ }^{26}{ }^{42}$ and the Borg 6-20 RPE scale may be used to assess and regulate upper-body physical activity at moderate-to-vigorous intensity in adults with chronic SCI. ${ }^{41}$ Moderate-intensity physical activity was selected as it is well tolerated, can be performed safely and is recommended for health risk reduction in individuals with SCI. ${ }^{2043}$

Participants will be permitted to work on a laptop computer, read, talk or watch DVDs during each condition. This will be standardised by asking participants to engage in the same activities during each of the two experimental conditions. Except during the activity bouts, participants will remain inactive and only leave their desk to void and consume standardised meals in a kitchen adjacent to the test laboratory; participants will be aided by a member of the research team when moving to these locations so that they remain inactive. A researcher will be present to ensure compliance with the protocols throughout all conditions.

\section{Meal and water consumption}

Standardised meals will be consumed immediately prior to the start of each experimental condition and at 3 hours, each providing $30 \%$ of estimated daily energy requirements for each participant. ${ }^{44}$ Participants will be asked to consume each meal within a $15 \mathrm{~min}$ time period. The time taken to consume the meals will be recorded for the first condition, and participants will be asked to replicate this time as closely as possible in the subsequent condition. Breakfast will consist of bran flakes, whole milk, croissant, butter and orange juice (54\% carbohydrate, $34 \%$ fat, $12 \%$ protein) and lunch will be a chicken sandwich, salted crisps and apple (54\% carbohydrate, $34 \%$ fat, $12 \%$ protein). The macronutrient composition of meals in the current study has been selected as it is generally representative of UK guidelines for a balanced diet. ${ }^{45}$ The glycaemic index for these breakfast and lunch meals is 43 and 72 , respectively. Glycaemic index values for each food item were obtained from the International Tables of Glycaemic Index and Glycaemic Load Values 2008, ${ }^{46}$ and meal glycaemic index was calculated using weighted means of the glycaemic index values for the component foods. ${ }^{47}$ Water will be available ad libitum during the first condition, and this volume of intake will be provided at standardised regular intervals in the subsequent condition.

\section{Blood collection and biochemistry}

Finger prick blood samples will be collected into two EDTA-containing microvettes (Microvette CB300 EDTA, Sarstedt, Leicester, UK) at baseline and at 30, 60, 90, 120, 180, 210, 240, 300 and $330 \mathrm{~min}$. Blood samples will be collected before the hourly activity bouts in SED-ACT. At each time point, approximately $600 \mu \mathrm{L}$ of whole blood will be collected. Blood glucose concentrations will be analysed immediately using the YSI 2300 STAT plus glucose and lactate analyzer (YSI, Yellow Springs, Ohio, USA) from $30 \mu \mathrm{L}$ of blood from one microvette. Additional $30 \mu \mathrm{L}$ volumes of whole blood will be aliquoted onto two separate Reflotron test strips (Roche Diagnostics, Burgess Hill, UK) for the measurement of triglyceride and HDL concentrations using the Reflotron Plus system (Roche Diagnostics, Burgess Hill, UK). The remaining whole blood $(\sim 490 \mu \mathrm{L})$ will be centrifuged at $2500 \mathrm{x}$ g for $5 \mathrm{~min}$ (Heraeus Pico 17, Thermo Scientific, Loughborough, UK) and the plasma then stored at $-80^{\circ} \mathrm{C}$. An ELISA kit will be used to determine plasma concentrations of insulin (Mercodia, Uppsala, Sweden).

\section{Blood pressure}

Blood pressure will be measured at baseline as described above followed by single readings taken at $60,120,180$, 240,300 and $330 \mathrm{~min}$. Readings will be taken $5 \mathrm{~min}$ before the hourly activity bouts in SED-ACT. Blood pressure will be measured using an automated oscillatory blood 
pressure monitor (Omron M5-I; Omron Matsusaka, Matsusaka, Japan).

\section{Study outcomes}

Primary outcome

The primary outcome for the study is within-participant, between condition postprandial glucose net incremental AUC (iAUC). ${ }^{11}$

\section{Secondary outcomes}

These include within-participant, between condition mean systolic and diastolic blood pressures, and net iAUC for postprandial triglycerides, HDL and insulin. Positive iAUC and total AUC will also be calculated for postprandial glucose, insulin, triglycerides and HDL to permit comparisons with previous studies.

\section{Feasibility measures}

To assess feasibility of the trial, participant dropout, number of experimental sessions completed, fatigue at the beginning and end of each day rated on an 11-point (0 'not fatigued at all' to 10 'extremely fatigued') Visual Analogue Scale (VAS) and the degree of difficulty in completing each experimental condition rated on an 11-point VAS (0 'not difficult at all' to 10 'extremely difficult') will be recorded. Participants will also complete the Physical Activity Enjoyment Scale ${ }^{48}$ at the end of the SED-ACT condition and report their enjoyment on a $200 \mathrm{~mm} \mathrm{VAS}^{49}$ ('Enjoyment') $20 \mathrm{~min}$ after the last activity bout in the SED-ACT condition. Participants will also report on the same scale how enjoyable they would find it to engage in this form of physical activity most days of the week in the coming month ('Expected enjoyment').

\section{Psychological outcomes}

Correlates of sedentary behaviour will be measured immediately before and after each experimental condition to explore whether participants' mood, affect, wellbeing and social cognitions regarding their ability to overcome being sedentary may differ in response to the SED-ACT condition compared with the SED condition. These measures will be based on the Theory of Planned Behaviour $^{50}$ using standardised wording formats ${ }^{51}$ that will include overcoming barriers (self-efficacy/perceived behavioural control), attitudes, intentions and action planning. The following questionnaires will be completed in this order: psychological well-being using the National Well-being Measurement ${ }^{52}$; the Warwick Edinburgh Mental Well-Being Scale ${ }^{53}$; current mood using the short Positive and Negative Affect Scale ${ }^{54}$; and an adapted version of the Schwarzer and Renner ${ }^{55}$ Physical Exercise Self-Efficacy Scale to measure self-efficacy to avoid long periods of sedentary time. These measures will be taken at the end of each experimental condition ( $330 \mathrm{~min}$ ) meaning that each questionnaire will be completed within $45 \mathrm{~min}$ following the last bout of activity in the SED-ACT condition. This is an appropriate time frame based on evidence that mood and affect are enhanced for 3-4hours following a single session of exercise. ${ }^{56}$
Although between-participant variation in the time taken to complete each questionnaire is anticipated, within-participant variation is expected to be limited, therefore permitting valid between-condition comparisons.

\section{Sample size calculations}

Sample size calculations were performed using GPower. ${ }^{57}$ Previous research reported a 16\% reduction (effect size, $F=0.61$ ) in 5-hour postprandial glucose total AUC when breaking up prolonged sedentary time with 2 min light-intensity walking every 20 min versus uninterrupted sitting in able-bodied participants. ${ }^{28}$ As this study will use arm cranking (localised muscular contractions) as opposed to walking where a larger muscle mass is required, a smaller effect may be observed. Based on this, it was estimated that 12 participants would be required for this complete two-treatment crossover design to detect a medium effect size $(F=0.4)$ with a within-person correlation of $0.6,80 \%$ power, and an $\alpha$ of 0.05 . To allow for potential withdrawals, a total of 18participants will be recruited.

\section{Statistical analysis}

Linear mixed models will be used to determine differences in the primary and secondary outcome variables between the conditions. All models will adjust for potential covariates explaining residual outcome variances (age, body fat $\%$, gender, lesion level, completeness of lesion and preprandial outcome values). Statistical significance will be accepted as $p \leq 0.05$. Cohen's d effect sizes will be calculated to describe the magnitude of differences between conditions.$^{58}$ Individuals' responses for CVD risk marker outcomes will also be compared between the conditions to determine the number of participants who respond to the experimental protocols.

\section{Patient and public involvement}

Patients and public were not involved with the development of the research question, outcome measures or study design, nor will they be involved with the conduct of the study. The recruitment plan was informed based on feedback from patients and public. A summary of the study results will be provided to each of the study participants.

\section{Ethics and dissemination}

Personal information about potential and enrolled participants will be stored in electronic format on password-protected computers or in hard copy format in locked filing cabinets at the University of Bedfordshire. Only members of the research team will have access to this information. All personal information will be destroyed after a period of 5 years. Individuals will be referred to in anonymised fashion in any published data.

The findings of this research will be disseminated to lay, academic, practice and policy-based audience via presentation at conference proceedings; publication in a peer-reviewed journal; websites, newsletters and social media; and summary reports to policy-makers and clinical care partners. The final trial dataset will be made available as supplementary material when the findings 
of the study are published in a peer-reviewed journal. Any protocol modifications will be communicated to the Cambridge South National Health Services Research Ethics Committee, recorded in the study's ISRCTN clinical trials registry and detailed in a journal publication of the study findings.

\section{Author affiliations}

${ }^{1}$ Institute for Sport and Physical Activity Research, School of Sport Science and Physical Activity, University of Bedfordshire, Bedford, UK

${ }^{2}$ School of Sport, Exercise and Health Sciences, The Peter Harrison Centre for Disability Sport, Loughborough University, Loughborough, UK ${ }^{3}$ Baker Heart and Diabetes Institute, Melbourne, Victoria, Australia ${ }^{4}$ Mary MacKillop Institute for Health Research, Australian Catholic University, Melbourne, Victoria, Australia

Acknowledgements The authors would like to thank Dr Jan van der Scheer for providing his advice on the design of the study protocol. The authors would also like to thank the patients and public who helped to inform the recruitment strategy for this study.

Contributors DPB and LC conceptualised the study. TMW, LC, VLG-T, DWD, CAL and DPB contributed to the design of the study protocol. TMW drafted the manuscript. TMW, LC, VLG-T, DWD, CAL and DPB commented and edited each section of the manuscript and approved the final version.

Funding This work is supported by Heart Research UK, grant number RG2655/17/18.

Disclaimer The funder has no role in the study design; collection, management, analysis and interpretation of data; writing of any reports; and the decision to submit any reports for publication, and will not have authority over any of these activities.

Competing interests None declared.

Patient consent Not required.

Ethics approval This study was approved on 19 May 2017 by the Cambridge South National Health Service Research Ethics Committee (reference 17/ $\mathrm{EE} / 0076)$.

Provenance and peer review Not commissioned; externally peer reviewed.

Open access This is an open access article distributed in accordance with the Creative Commons Attribution Non Commercial (CC BY-NC 4.0) license, which permits others to distribute, remix, adapt, build upon this work non-commercially, and license their derivative works on different terms, provided the original work is properly cited and the use is non-commercial. See: http://creativecommons.org/ licenses/by-nc/4.0/

(C) Article author(s) (or their employer(s) unless otherwise stated in the text of the article) 2018. All rights reserved. No commercial use is permitted unless otherwise expressly granted.

\section{REFERENCES}

1. Lee BB, Cripps RA, Fitzharris $M$, et al. The global map for traumatic spinal cord injury epidemiology: update 2011 , global incidence rate. Spinal Cord 2014;52:110-6.

2. Royal College of Physician. British Society of Rehabilitation Medicine, Chronic spinal cord injury: management of patients in acute hospital settings: National Guidelines. London, UK: Royal College of Physician, 2008. (accessed 26 Apr 2016).

3. Garshick E, Kelley A, Cohen SA, et al. A prospective assessment of mortality in chronic spinal cord injury. Spinal Cord 2005:43:408-16.

4. Cragg JJ, Noonan VK, Krassioukov A, et al. Cardiovascular disease and spinal cord injury: results from a national population health survey. Neurology 2013;81:723-8.

5. Gorgey AS, Dolbow DR, Dolbow JD, et al. Effects of spinal cord injury on body composition and metabolic profile - part I. J Spinal Cord Med 2014;37:693-702.

6. Bauman WA, Spungen AM. Disorders of carbohydrate and lipid metabolism in veterans with paraplegia or quadriplegia: a model of premature aging. Metabolism 1994;43:749-56.
7. Wahman K, Nash MS, Westgren N, et al. Cardiovascular disease risk factors in persons with paraplegia: the Stockholm spinal cord injury study. J Rehabil Med 2010;42:272-8.

8. Ford ES, Giles WH, Dietz WH. Prevalence of the metabolic syndrome among US adults: findings from the third National Health and Nutrition Examination Survey. JAMA 2002;287:356-9.

9. Gater DR. Obesity after spinal cord injury. Phys Med Rehabil Clin N Am 2007;18:333-51.

10. Gorgey AS, Dolbow DR, Dolbow JD, et al. The effects of electrical stimulation on body composition and metabolic profile after spinal cord injury--Part II. J Spinal Cord Med 2015;38:23-37.

11. O'Keefe JH, Bell DS. Postprandial hyperglycemia/hyperlipidemia (postprandial dysmetabolism) is a cardiovascular risk factor. $\mathrm{Am} \mathrm{J}$ Cardiol 2007;100:899-904.

12. Esposito K, Giugliano D, Nappo F, et al. Regression of carotid atherosclerosis by control of postprandial hyperglycemia in type 2 diabetes mellitus. Circulation 2004;110:214-9.

13. Smith NL, Barzilay JI, Shaffer D, et al. Fasting and 2-hour postchallenge serum glucose measures and risk of incident cardiovascular events in the elderly: the Cardiovascular Health Study. Arch Intern Med 2002;162:209-16.

14. Duckworth WC, Jallepalli P, Solomon SS. Glucose intolerance in spinal cord injury. Arch Phys Med Rehabil 1983;64:107-10.

15. Bauman WA, Adkins RH, Spungen AM, et al. The effect of residual neurological deficit on oral glucose tolerance in persons with chronic spinal cord injury. Spinal Cord 1999;37:765-71.

16. Raymond J, Harmer AR, Temesi J, et al. Glucose tolerance and physical activity level in people with spinal cord injury. Spinal Cord 2010;48:591-6.

17. Bauman WA, Adkins RH, Spungen AM, et al. The effect of residual neurological deficit on serum lipoproteins in individuals with chronic spinal cord injury. Spinal Cord 1998;36:13-17.

18. Nash MS, DeGroot J, Martinez-Arizala A, et al. Evidence for an exaggerated postprandial lipemia in chronic paraplegia. J Spinal Cord Med 2005;28:320-5.

19. Groah SL, Nash MS, Ljungberg IH, et al. Nutrient intake and body habitus after spinal cord injury: an analysis by sex and level of injury. J Spinal Cord Med 2009;32:25-33.

20. Martin Ginis KA. Evidence-based scientific exercise guidelines for adults with spinal cord injury: an update and a new guideline, 2017.

21. Ginis KA, Arbour-Nicitopoulos KP, Latimer AE, et al. Leisure time physical activity in a population-based sample of people with spinal cord injury part II: activity types, intensities, and durations. Arch Phys Med Rehabil 2010;91:729-33.

22. Buchholz AC, Martin Ginis KA, Bray SR, et al. Greater daily leisure time physical activity is associated with lower chronic disease risk in adults with spinal cord injury. Appl Physiol Nutr Metab 2009;34:640-7.

23. Ginis KA, Hicks AL, Latimer AE, et al. The development of evidenceinformed physical activity guidelines for adults with spinal cord injury. Spinal Cord 2011;49:1088-96.

24. Tremblay MS, Aubert S, Barnes JD, et al. Sedentary Behavior Research Network (SBRN) - Terminology consensus project process and outcome. Int J Behav Nutr Phys Act 2017:14:75.

25. Dunstan DW, Thorp AA, Healy GN. Prolonged sitting: is it a distinct coronary heart disease risk factor? Curr Opin Cardiol 2011;26:412-9.

26. Dunstan DW, Kingwell BA, Larsen R, et al. Breaking up prolonged sitting reduces postprandial glucose and insulin responses. Diabetes Care 2012;35:976-83.

27. Miyashita M, Edamoto K, Kidokoro T, et al. Interrupting sitting time with regular walks attenuates postprandial triglycerides. Int J Sports Med 2016;37:97-103.

28. Bailey DP, Locke CD. Breaking up prolonged sitting with lightintensity walking improves postprandial glycemia, but breaking up sitting with standing does not. J Sci Med Sport 2015;18:294-8.

29. Larsen RN, Kingwell BA, Sethi P, et al. Breaking up prolonged sitting reduces resting blood pressure in overweight/obese adults. Nutr Metab Cardiovasc Dis 2014;24:976-82.

30. Chan AW, Tetzlaff JM, Altman DG, et al. SPIRIT 2013 statement: defining standard protocol items for clinical trials. Ann Intern Med 2013;158:200-7.

31. New PW, Marshall R. International Spinal Cord Injury Data Sets for non-traumatic spinal cord injury. Spinal Cord 2014;52:123-32.

32. Jacobs PL, Nash MS. Exercise recommendations for individuals with spinal cord injury. Sports Med 2004;34:727-51.

33. Ekelund U, Steene-Johannessen J, Brown WJ, et al. Does physical activity attenuate, or even eliminate, the detrimental association of sitting time with mortality? A harmonised meta-analysis of data from more than 1 million men and women. Lancet 2016;388:1302-10.

34. Keil M, Totosy de Zepetnek JO, Brooke-Wavell K, et al. Measurement precision of body composition variables in elite wheelchair 
athletes, using dual-energy X-ray absorptiometry. Eur J Sport Sci 2016;16:65-71.

35. Spungen AM, Adkins RH, Stewart CA, et al. Factors influencing body composition in persons with spinal cord injury: a cross-sectional study. J Appl Physiol 2003;95:2398-407.

36. Gorgey AS, Dolbow DR, Gater DR. A model of prediction and crossvalidation of fat-free mass in men with motor complete spinal cord injury. Arch Phys Med Rehabil 2012;93:1240-5.

37. Marfell-Jones MJ, Stewart AD, De Ridder JH. International standards for anthropometric assessment. 2012.

38. Willems A, Paulson TA, Keil M, et al. Dual-Energy X-Ray Absorptiometry, skinfold thickness, and waist circumference for assessing body composition in ambulant and non-ambulant wheelchair games players. Front Physiol 2015;6.

39. Borg GA. Psychophysical bases of perceived exertion. Med Sci Sports Exerc 1982;14:377???381-81.

40. Bailey DP, Maylor BD, Orton CJ, et al. Effects of breaking up prolonged sitting following low and high glycaemic index breakfast consumption on glucose and insulin concentrations. Eur J Appl Physiol 2017;117:1299-307.

41. van der Scheer JW, Hutchinson MJ, Paulson T, et al. Reliability and Validity of Subjective Measures of Aerobic Intensity in Adults With Spinal Cord Injury: A Systematic Review. Pm R 2018;10.

42. Bailey DP, Broom DR, Chrismas BC, et al. Breaking up prolonged sitting time with walking does not affect appetite or gut hormone concentrations but does induce an energy deficit and suppresses postprandial glycaemia in sedentary adults. Appl Physiol Nutr Metab 2016;41:324-31.

43. Burr JF, Shephard RJ, Zehr EP. Physical activity after stroke and spinal cord injury: evidence-based recommendations on clearance for physical activity and exercise. Can Fam Physician 2012;58:1236-9.

44. Schofield WN. Predicting basal metabolic rate, new standards and review of previous work. Hum Nutr Clin Nutr 1985;39(Suppl 1):5-41.

45. Food Standards Agency. FSA nutrient and food based guidelines for UK institutions. 2007 http://www.food.gov.uk/sites/default/files/ multimedia/pdfs/nutrientinstitution.pdf (accessed 18 Jan 2016).
46. Atkinson FS, Foster-Powell K, Brand-Miller JC. International tables of glycemic index and glycemic load values: 2008. Diabetes Care 2008;31:2281-3.

47. Wolever TM, Jenkins DJ. The use of the glycemic index in predicting the blood glucose response to mixed meals. Am J Clin Nutr 1986;43:167-72.

48. Kendzierski D, DeCarlo KJ. Physical Activity Enjoyment Scale: Two Validation Studies. Journal of Sport and Exercise Psychology 1991;13:50-64.

49. Svensson E. Comparison of the Quality of Assessments Using Continuous and Discrete Ordinal Rating Scales. Biometrical Journal 2000;42:417-34.

50. Ajzen I. The theory of planned behavior. Organ Behav Hum Decis Process 1991;50:179-211.

51. Francis J. Constructing questionnaires based on the theory of planned behaviour: a manual for health services researchers. Newcastle upon Tyne, UK: Centre for Health Services Research, University of Newcastle upon Tyne, 2004.

52. Tinkler L. Measuring National Well-being: Personal Well-being in the UK, 2014 to 2015. 2015 https://www.ons.gov.uk/peoplepopula tionandcommunity/wellbeing/bulletins/measuringnationalwellbeing/ 2015-09-23.

53. Tennant R, Hiller L, Fishwick R, et al. The Warwick-Edinburgh Mental Well-being Scale (WEMWBS): development and UK validation. Health Qual Life Outcomes 2007;5:63.

54. Thompson ER. Development and validation of an internationally reliable short-form of the positive and negative affect schedule (PANAS). J Cross Cult Psychol 2007;38:227-42.

55. Schwarzer R, Renner B. Social-cognitive predictors of health behavior: action self-efficacy and coping self-efficacy. Health Psychol 2000;19:487-95.

56. Yeung RR. The acute effects of exercise on mood state. $J$ Psychosom Res 1996;40:123-41.

57. Faul F, Erdfelder E, Lang AG, et al. G*Power 3: a flexible statistical power analysis program for the social, behavioral, and biomedical sciences. Behav Res Methods 2007;39:175-91.

58. Cohen J. Statistical power analysis for the behavioral sciences. 2nd ed. Hillsdale, NJ: Erlbaum, 1988. 